\title{
Oral Health Status in the Pars Cohort Study
}

\author{
Saba Ghazimoghadam, MD, MPHㅜ; Alireza Salehi, MD, MPH, PhD ${ }^{2 *}$, Hossein Molavi Vardanjani, MPH, PhD; Fereshte Shafiei, \\ DMD, MSc ${ }^{3}$; Peyman Arasteh, MD, MPH${ }^{1}$; Abdullah Gandomkar, MD ${ }^{4}$; Hossein Poustchi, MD, PhD ; Reza Malekzadeh, MD ${ }^{4,5}$ \\ ${ }^{1}$ Department of MPH, Shiraz University of Medical Sciences, Shiraz, Iran \\ ${ }^{2}$ Shiraz University of Medical Sciences, Shiraz, Iran \\ ${ }^{3}$ Oral and Dental Disease Research Center, Department of Operative Dentistry, School of Dentistry, Shiraz University of Medical \\ Sciences, Shiraz, Iran \\ ${ }^{4}$ Non-Communicable Disease Research Center, Shiraz University of Medical Sciences, Shiraz, Iran \\ ${ }^{5}$ Liver, Pancreatic and Biliary Diseases Research Center, Digestive Disease Research Institute, Tehran University of Medical Sciences, \\ Tehran, Iran
}

\begin{abstract}
Background: Poor oral health is common in the world, especially in low-income and developing countries. We evaluated oral health status and its association with sociodemographic and economic variables in one of the largest cohort studies in Iran. Methods: This cross-sectional study was conducted using data from the Pars Cohort Study which is conducted in a rural area in southern Iran. A total of 9264 individuals were enrolled in the initial registry. Data on sociodemographic and clinical characteristics were collected. Oral assessment was completed by trained physicians. Generalized negative binomial regression modeling was applied.

Results: Overall, data from 9264 participants entered the final analysis. The participants' mean age was $52.6 \pm 9.7$ years. In total, 8975 participants $(96.8 \%)$ had at least one tooth loss and 1790 participants $(19.3 \%)$ were edentulous. Female gender (incidence rate ratio [IRR]: 1.05, 95\% Cl: 1.02, 1.08) (IRR: 1.12, 95\% Cl: 1.07, 1.16), opium consumption (IRR: 1.17, 95\% Cl: 1.12, 1.22) (IRR: 1.23, 95\% Cl: 1.16 1.30), lower socioeconomic status (SES) (IRR: 1.07, 95\% Cl: 1.03, 1.10) (IRR: 1.10, 95\% Cl: 1.06, 1.15) and being illiterate (IRR: 1.23, 95\% Cl: 1.20, 1.26) (IRR: 1.36, 95\% Cl: 1.32, 1.41) were associated with higher decayed, missing and filled teeth (DMFT) and more tooth loss.

Conclusion: Oral health was poor in our study sample, especially among older individuals, people with lower SES, illiterate participants, smokers and opiate consumers, which shows the need for improvement in oral health care.

Keywords: DMF Index, Iran, Oral health, Pars Cohort Study, Risk factors, Tooth loss

Cite this article as: Ghazimoghadam S, Salehi A, Molavi Vardanjani H, Shafiei F, Arasteh P, Gandomkar A, et al. Oral health status in the pars cohort study. Arch Iran Med. 2021;24(4):273-279. doi: 10.34172/aim.2021.38
\end{abstract}

Received: October 31, 2020, Accepted: November 4, 2020, ePublished: April 1, 2021

\section{Introduction}

According to the World Dental Federation (Fédération Dentaire Internationale; FDI), oral health is multifaceted and includes the ability to speak, smile, smell, taste, touch, chew, swallow, and conveys a range of emotions through facial expressions with confidence and without pain, discomfort, and disease of the craniofacial complex. ${ }^{1}$ Poor oral health, including periodontal disease and tooth loss, is common all over the world. In 2010, 2.3\% of the world population (158 million people) were edentate. ${ }^{2}$ Furthermore, dental caries is the most common oral disease worldwide and almost every person experiences caries during their life time. ${ }^{3}$ According to the World Health Organization (WHO), oral disease stands as the fourth most expensive chronic disease to treat. ${ }^{4}$

Poor oral hygiene can result in serious comorbidities including periodontitis, pain, speech and psychological problems, tooth decay, loss of tooth and discomfort in gums or teeth. ${ }^{5}$ Epidemiologic studies have shown the association between periodontal disease, tooth loss and dental caries with an increased risk of chronic diseases such as cardiovascular disease and diabetes and also higher risk of cancer at different sites including lungs, oral cavity, esophagus, stomach and pancreas. ${ }^{6}$ Due to the growing elderly population, the prevalence rates of oral and dental diseases are increasing. ${ }^{2}$ Total or partial edentulism may be the onset of disability and mortality, particularly in the elderly. ${ }^{7}$

Many factors affect oral health, including socioeconomic and environmental status, income, urbanization and access to health services. ${ }^{8}$ Excessive and frequent consumption of sugar, inadequate fluoride exposure, and smoking and tobacco use are associated with increased incidence of dental caries and periodontal diseases. ${ }^{9}$

Oral diseases pose the greatest burden on the poor and socially marginalized people. ${ }^{10}$ In Iran, a developing country that has experienced an increase in the rate of older individuals, oral health has become a major public health issue. ${ }^{11}$ According to different reports from epidemiological studies, ${ }^{12-14}$ and mainly due to a lack in 
nationwide registries, data on oral health in many regions is scarce and largely missing, especially in middle- and low-income regions including southern Iran.

Thus, in this study, we aim to determine the oral health status and its association with sociodemographic and economic variables in one of the largest cohort studies conducted in southern Iran.

\section{Materials and Methods \\ Study Design and Settings}

This is a cross-sectional study using data from the Pars Cohort Study (PCS). The PCS is an ongoing cohort study which started in 2012 in Valashahr district, a rural area consisting of Valashahr city and 93 villages in southern Iran, home to more than 40000 inhabitants. In total, 9721 participants aged 40 to 75 years were invited and 9264 of them were recruited from 2012 to 2014 . Potential participants were excluded if they were unwilling to participate or were a temporary resident. More details on the PCS design have been published previously. ${ }^{15}$

The primary aim of the cohort study was to perform an in-depth investigation of non-communicable diseases. Accordingly, a list of more than 200 variables including socio-demographic, clinical and laboratory data were collected from each individual in the initial phase of the cohort. This research was approved by the Ethics Committees of both Tehran and Shiraz Universities of Medical Sciences. The purpose of the study was explained to the participants and informed written consents were obtained.

\section{Oral Health Assessment}

A structured questionnaire was designed which contained questions about oral health. First, the participants were asked if they had lost any permanent teeth, and if their answer was yes, the age at which the first permanent tooth was lost was inquired. Participants were also asked regarding removable partial or full dentures and whether they wore these dentures during the day. Frequency of tooth brushing was reported as never, once a day, twice a day, three times a day and other (which was less than once daily). Before starting the study, physicians were trained by a dentist. The physicians determined decayed, missing and filled teeth (DMFT) visually and used dental probes to assess cavitation.

\section{Data Preparation}

Data cleaning was done using appropriate techniques. ${ }^{16}$ Inconsistent or erroneous data were identified and corrected. Data on age, sex and ethnicity (Persian, Turk and other ethnicities) were acquired. Educational level was categorized in 3 classes (illiterate, less than diploma and university degree). The multiple correspondence analysis (MCA) technique was applied and accordingly, the socioeconomic status (SES) of each participant was estimated. The participants were categorized into four classes (quartiles of estimated latent factor by MCA) including low, middle-low, middle-high, and high SES. ${ }^{17}$ History of cigarette use, current use of cigarettes, use of tobacco products other than cigarettes and history of opium use were taken. For physical activity, the participants were classified into three groups (low, medium and high) based on their metabolic equivalent of task (MET) score. ${ }^{18}$ Dental health indices including DMFT, missing teeth (MT), filled teeth (FT), decayed teeth (DT), and age at first tooth loss were categorized into four categories (quartiles), where distribution was inflated on a single value (i.e. 32 for MT, or zero for FT/DT), a separate category was defined for that value, and quartiles were defined after removing that value. Cases with tooth brushing twice or three times a day were put in a single category due to their small numbers. Finally, dental health indices for elderly people (65-75 years old) were also assessed.

\section{Statistical Analysis}

Data was described as frequency and percentage for descriptive variables and as mean and standard deviations and as median and interquartile range for quantitative variables.

Rate of DMFT and MT was calculated by dividing the value of each variable by 32 (the total number of teeth). The denominator for calculating the rate of DT or FT was the number of remaining teeth. Data analyses were done assuming a Poisson distribution for these rates. Because of a significant over-dispersion and also inflation of the distribution of dental health indices (i.e. DMFT, DT, MT, FT), data was analyzed using univariate and multivariable Generalized Negative Binomial regression (GNBReg). Four GNBReg models were fitted. Each model assessed the correlates of each of the four dental health indices. Crude and adjusted incidence rate ratios (IRR) and their 95\% confidence intervals (CI) were estimated. Variables with likelihood ratio test $P$ values of less than 0.1 were retained in the multivariate logistic regression model. $P$ values less than 0.05 were considered significant. Data was analyzed using the Stata software (Release 11; StataCorp. College station, TX, USA)

\section{Results}

Overall, 9264 participants entered the final analysis. The participants' mean (SD) age was 52.6 (9.7) years and $53.8 \%$ of them were females. Other sociodemographic characteristics of participants are shown in Table S1 of Supplementary file 1.

The mean (SD) number of missing, decayed, and filled teeth were 15.2 (10.6), 3.37 (3.9), and 0.5 (1.5), respectively. The mean (SD) DMFT index was 19.1 (9.5). Overall, 8975 participants $(96.8 \%)$ had at least one tooth loss (4145 [96.9\%] men and 4829 [96.7\%] women). The proportion of edentulous people was 19.3\%. Among 
these, $6.4 \%$ had no dentures. In the senior age group $(\geq 65$ years old), $43.7 \%$ were edentulous among whom $8.9 \%$ had no dentures. The mean (SD) age at first tooth loss was 24.2 (11.7) years (24.6 [11.7] for men and 23.8 [11.8] for women) $(P$ value $<0.05)$. The prevalence of having at least one tooth decay in our total participants and in seniors was $68.9 \%$ and $50.3 \%$, respectively. In Table 1 , the mean DMFT in different groups is reported.

In general, 4393 (47.4\%) individuals did not brush their teeth at all, 2240 participants (24.2\%) less than daily, 2037 participants $(22.0 \%)$ brushed their teeth daily, and 592 participants $(6.4 \%)$ brushed their teeth 2 or 3 times a day. Regarding ethnicity, $44.1 \%$ of Persians and $55.9 \%$ of non-Persian participants did not brush their teeth at all. Women showed a higher prevalence of tooth loss than men

Table 1. Mean of DMFT in Different Groups*

\begin{tabular}{|c|c|c|c|}
\hline Variables & No. $(\%)$ & Men & Women \\
\hline Marital status & & $P=0.106$ & $P<0.001$ \\
\hline Never married & $297(3.2)$ & $17.9 \pm 8.9$ & $14.2 \pm 8.9$ \\
\hline Married & $8210(88.7)$ & $18.8 \pm 9.5$ & $19.0 \pm 9.5$ \\
\hline Widowed or divorced & & $21.8 \pm 9.7$ & $22.9 \pm 9.0$ \\
\hline Ethnicity & $752(8.2)$ & $P<0.001$ & $P<0.001$ \\
\hline Persian & $5215(56.3)$ & $18.2 \pm 9.8$ & $18.6 \pm 9.9$ \\
\hline Non-Persian & & $19.6 \pm 9.0$ & $20.3 \pm 8.9$ \\
\hline Education class & 4047 (43.7) & $P<0.001$ & $P<0.001$ \\
\hline Illiterate & 4538 (49.0) & $22.8 \pm 8.7$ & $21.9 \pm 9.2$ \\
\hline Less than diploma & $443647.9)$ & $17.5 \pm 9.3$ & $14.7 \pm 8.4$ \\
\hline University degree & & $13.4 \pm 7.7$ & $13.2 \pm 7.7$ \\
\hline Cigarette ever used & $281(3.0)$ & $P<0.001$ & $P=0.461$ \\
\hline No & $7345(79.3)$ & $17.2 \pm 9.2$ & $19.3 \pm 9.6$ \\
\hline Yes & & $21.0 \pm 9.4$ & $20.4 \pm 9.8$ \\
\hline Cigarette current use & $1917(20.7)$ & $P<0.001$ & $P=0.991$ \\
\hline No & $7953(86.0)$ & $17.7 \pm 9.4$ & $19.3 \pm 9.6$ \\
\hline Yes & & $21.6 \pm 9.1$ & $19.3 \pm 10.2$ \\
\hline Opiate ever used & $1296(14.0)$ & $P<0.001$ & $P<0.001$ \\
\hline No & 8488 (91.6) & $18.0 \pm 9.4$ & $19.3 \pm 9.6$ \\
\hline Yes & & $22.7 \pm 8.8$ & $21.8 \pm 10.6$ \\
\hline Tobacco ever used & $774(8.4)$ & $P<0.001$ & $P<0.001$ \\
\hline No & $5626(62.0)$ & $18.0 \pm 9.5$ & $18.2 \pm 9.5$ \\
\hline Yes & & $20.8 \pm 9.3$ & $20.6 \pm 9.5$ \\
\hline Physical activity & 3443 (38.0) & $P=0.199$ & $P=0.002$ \\
\hline Low & 3060 (33.0) & $18.5 \pm 10.0$ & $19.2 \pm 9.9$ \\
\hline Medium & 3056 (33.0) & $18.7 \pm 9.7$ & $18.9 \pm 9.5$ \\
\hline High & & $19.1 \pm 9.1$ & $20.2 \pm 8.9$ \\
\hline SES & $3146(34.0)$ & $P<0.001$ & $P<0.001$ \\
\hline Low & $2408(26.1)$ & $20.7 \pm 9.2$ & $21.3 \pm 9.2$ \\
\hline Middle-low & $2490(27.0)$ & $20.1 \pm 9.3$ & $20.2 \pm 9.5$ \\
\hline Middle-high & $2043(22.1)$ & $18.4 \pm 9.5$ & $18.1 \pm 9.6$ \\
\hline High & $2291(24.8)$ & $16.6 \pm 9.4$ & $16.7 \pm 9.4$ \\
\hline
\end{tabular}

DMFT, missing and filled teeth; SES, socioeconomic status.

*Data are presented as mean and standard deviation. (mean 15.8; SD 10.9 vs. mean 14.5; SD 10.1, $P<0.001$ ). Oral health determinants (DMFT and its subgroups, upper and lower denture use, frequency of tooth brushing and age at first tooth loss) are shown in Table 2.

In the negative binomial regression model, factors associated with tooth loss were: increasing age (IRR: 1.52, 95\% CI: 1.46, 1.58), female gender (IRR: 1.12, 95\% CI: $1.07,1.16$ ), less than daily brushing (IRR: $1.06,95 \% \mathrm{CI}$ : $1.03,1.10$ ), opium consumption (IRR: 1.23 , 95\% CI: $1.16,1.30)$, previous or current cigarette consumption (IRR: $1.17,95 \%$ CI: $1.10,1.25$ ) (IRR: $1.14,95 \%$ CI: $1.06,1.21$ ), tobacco consumption (IRR: $1.11,95 \% \mathrm{CI}$ : $1.08,1.15$ ), ethnicity other than Persian (IRR: 1.03, 95\% CI: 1.00, 1.06), lower SES (IRR: 1.10, 95\% CI: 1.06, 1.15) and being illiterate (IRR: 1.36, 95\% CI: 1.32, 1.41). The results for DMFT, tooth decay and filled teeth are shown in Table 3 (Table of crude IRR is shown in Table S1 of Supplementary file 1).

\section{Discussion}

In our study, almost everyone had lost at least one tooth $(96.8 \%)$ and less than one percent of the population had preserved all of their teeth. This represents the undesirable status of oral health in the studied population.

The prevalence of edentulism in our whole population was 19\%. Peltzer and colleagues evaluated the prevalence of loss of all teeth among persons 50 years and older in 6 countries. ${ }^{19}$ Their findings showed the prevalence rate in different countries to be at $3.0 \%$ in Ghana, $8.5 \%$ in South Africa, $9.0 \%$ in China, $16.3 \%$ in India, $18.0 \%$ in the Russian Federation and $21.7 \%$ in Mexico. According to a report by the National Center for Health Statistics, ${ }^{20}$ approximately $19 \%$ of people aged 65 years or older in the United States were edentulous. In comparison, in our senior population, $43 \%$ had no natural teeth. Edentulous people tend to eat less fresh fruits and vegetables which results in deterioration in their nutrition and health. Furthermore, it leads to social isolation and loneliness due to embarrassment and loss of self-esteem. ${ }^{21}$

The prevalence of dental caries reported in our total participants and in seniors was $68.9 \%$ and $50.3 \%$, respectively, which is much more than that reported in other regions of the world. The prevalence of root caries among individuals within a senior age group (65-74-yearold) was $14.4 \%$ in Spain, ${ }^{13} 24.0 \%$ in Belgium, ${ }^{13} 28 \%$ in Germany, ${ }^{13} 28.4 \%$ in Turkey, ${ }^{22}$ and $38.3 \%$ in Greece. ${ }^{23}$

We found that women had more tooth loss than men ( $15.8 \%$ vs. $14.5 \%, P$ value $<0.001)$ which is similar to the figures of other reports. ${ }^{24,25}$ This can be attributed to the bio-psychological conditions women face during their life; multiple births and breast feeding which remarkably affect their oral and dental health. ${ }^{11}$

The rate of tooth loss (IRR: 1.52) and the rate of decayed teeth (IRR: 1:54) in our population increased with increasing age, which was expected as physiological 
Table 2. Oral Health Indices by Age and Sex*

\begin{tabular}{|c|c|c|c|c|c|c|}
\hline \multirow{2}{*}{ Variables } & \multirow{2}{*}{$n(\%)$} & \multicolumn{2}{|c|}{ Male } & \multicolumn{2}{|c|}{ Female } & \multirow{2}{*}{$P$ value } \\
\hline & & $40-59$ years & $\geq 60$ years & $40-59$ years & $\geq 60$ years & \\
\hline DMFT & & \multicolumn{2}{|c|}{$P<0.001$} & \multicolumn{2}{|c|}{$P<0.001$} & \multirow{6}{*}{0.014} \\
\hline Category $1(\leq 10)$ & $2086(22.5)$ & $928(28.3)$ & $89(9.0)$ & $981(26.1)$ & $88(7.1)$ & \\
\hline Category 2 (11-15) & $1777(19.2)$ & $701(21.4)$ & $101(10.2)$ & 859 (22.9) & $116(9.4)$ & \\
\hline Category 3 (16-22) & 1847 (19.9) & $671(20.5)$ & $165(16.6)$ & $842(22.4)$ & $169(13.7)$ & \\
\hline Category 4 (23-31) & $1526(16.5)$ & $547(16.7)$ & $256(25.8)$ & $463(12.3)$ & $260(21.1)$ & \\
\hline Category 5 (32) & $2023(21.8)$ & $433(13.2)$ & $382(35.8)$ & $609(16.2)$ & $599(48.6)$ & \\
\hline Number of teeth lost & & \multicolumn{2}{|c|}{$P<0.001$} & \multicolumn{2}{|c|}{$P<0.001$} & \multirow{6}{*}{$<0.001$} \\
\hline Category $1(\leq 5)$ & $1915(20.7)$ & $855(26.1)$ & $69(6.95)$ & $916(24.4)$ & $75(6.1)$ & \\
\hline Category 2 (6-10) & $2054(22.2)$ & $863(26.3)$ & $134(15.3)$ & $943(25.1)$ & $119(9.7)$ & \\
\hline Category 3 (11-16) & $1779(19.2)$ & $680(20.7)$ & $111(12.7)$ & $765(20.4)$ & $169(13.7)$ & \\
\hline Category 4 (17-31) & 1725 (18.6) & $561(17.1)$ & $305(30.7)$ & $548(14.6)$ & $311(25.2)$ & \\
\hline Category 5 (32) & $1789(19.3)$ & $321(9.8)$ & 327 (32.9) & $582(15.5)$ & $559(45.3)$ & \\
\hline Number of teeth decayed & & \multicolumn{2}{|c|}{$P<0.001$} & \multicolumn{2}{|c|}{$P<0.001$} & \multirow{6}{*}{$<0.001$} \\
\hline Category $1(0)$ & $2873(31.0)$ & $710(21.6)$ & $400(40.2)$ & $1117(29.8)$ & $646(52.4)$ & \\
\hline Category $2(1-2)$ & $1959(21.2)$ & 769 (23.4) & $166(16.7)$ & $842(22.4)$ & $182(14.8)$ & \\
\hline Category 3 (3-4) & $1806(19.5)$ & $665(20.3)$ & 158 (15.9) & $831(22.1)$ & $152(12.3)$ & \\
\hline Category 4 (5-6) & $1113(12.0)$ & $423(12.9)$ & $97(9.7)$ & $477(12.7)$ & $116(9.4)$ & \\
\hline Category $5(\geq 7)$ & $1511(16.3)$ & $713(21.7)$ & $174(17.5)$ & $487(13.0)$ & $137(11.1)$ & \\
\hline Number of teeth filled & & \multicolumn{2}{|c|}{$P<0.001$} & \multicolumn{2}{|c|}{$P<0.001$} & \multirow{6}{*}{0.841} \\
\hline Category $1(0)$ & 7774 (83.9) & $2629(80.2)$ & $944(94.9)$ & $3012(80.2)$ & $1189(96.4)$ & \\
\hline Category 2 (1) & $417(4.5)$ & $176(5.4)$ & $22(2.2)$ & $205(5.5)$ & $14(1.1)$ & \\
\hline Category $3(2-3)$ & $534(5.8)$ & $252(7.7)$ & $15(1.5)$ & $247(6.6)$ & $20(1.6)$ & \\
\hline Category 4 (4) & $184(2.0)$ & $81(2.5)$ & $7(0.7)$ & $93(2.5)$ & $3(0.2)$ & \\
\hline Category $5(\geq 5)$ & $353(3.8)$ & $142(4.3)$ & $7(0.7)$ & $197(5.2)$ & $7(0.6)$ & \\
\hline Age at first tooth loss & & \multicolumn{2}{|c|}{$P<0.001$} & \multicolumn{2}{|c|}{$P<0.001$} & \multirow{6}{*}{$<0.001$} \\
\hline Age at first loss category $1(\geq 31)$ & $1232(13.3)$ & $483(14.7)$ & $160(16.1)$ & $432(11.5)$ & $157(12.7)$ & \\
\hline Age at first loss category $2(25-30)$ & $2410(26.0)$ & $825(25.2)$ & $343(34.5)$ & $904(24.1)$ & $338(27.4)$ & \\
\hline Age at first loss category $3(21-24)$ & $864(9.3)$ & $331(10.1)$ & $75(7.5)$ & $342(9.1)$ & $116(9.4)$ & \\
\hline Age at first loss category 4 (18-20) & $2732(29.5)$ & $932(28.4)$ & 257 (25.9) & $1176(31.3)$ & $367(29.8)$ & \\
\hline Age at first loss category $5(\leq 17)$ & $2019(21.8)$ & 707 (21.6) & $159(16.0)$ & $888(23.9)$ & $255(20.7)$ & \\
\hline Frequency of tooth brushing & & \multicolumn{2}{|c|}{$P=0.300$} & \multicolumn{2}{|c|}{$P=0.021$} & \multirow{5}{*}{0.496} \\
\hline Never & $4319(47.6)$ & $1546(47.2)$ & $423(47.9)$ & $1799(48.0)$ & $551(47.3)$ & \\
\hline Other (less than daily) & $2236(24.6)$ & $853(26.0)$ & $213(24.1)$ & $894(23.8)$ & $276(23.7)$ & \\
\hline Once a day & $1929(21.3)$ & $676(20.6)$ & $181(20.5)$ & $828(22.1)$ & 244 (20.9) & \\
\hline Two or more times a day & $592(6.6)$ & $201(6.1)$ & $60(6.8)$ & $229(6.1)$ & $95(8.1)$ & \\
\hline Hoving unnon dontun & $2277(260)$ & & & & & 0001 \\
\hline graving upper uenture & 251 (20.2) & $481(14.7)$ & $292(33.0)$ & $1023(27.3)$ & $581(49.8)$ & -0.001 \\
\hline & & & & & & \\
\hline naving lower denture & $2141(23.0)$ & $416(12.7)$ & 282 (31.9) & $886(23.6)$ & $557(47.7)$ & $<0.001$ \\
\hline Hnnon dontun & (2020 & & & & & \\
\hline Opper aenture use & $2320(25.6)$ & $471(14.4)$ & 277 (31.3) & $1006(26.8)$ & $566(48.5)$ & $<0.001$ \\
\hline Iower denture use & $2103(230)$ & & & & & \\
\hline 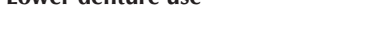 & $2100(23.2)$ & 407 (12.4) & $272(30.8)$ & 877 (23.4) & 547 (46.9) & -0.00 \\
\hline
\end{tabular}

*Data are presented as frequency and percentage. 
Table 3. Generalized Negative Binominal Regression Analysis of Factors Associated with DMFT, Tooth Loss, Tooth Decay and Filled Teeth in Pars Cohort Study*

\begin{tabular}{|c|c|c|c|c|}
\hline Variable & DMFT & Missing teeth & Decayed teeth $^{* *}$ & Filled teeth $^{* *}$ \\
\hline \multicolumn{5}{|l|}{ Age } \\
\hline 40-59 years & Ref & Ref & Ref & Ref \\
\hline$\geq 60$ years & $1.34(1.31,1.38)$ & $1.52(1.46,1.58)$ & $1.54(1.41,1.68)$ & $0.70(0.56,0.88)$ \\
\hline \multicolumn{5}{|l|}{ Gender } \\
\hline Male & Ref & Ref & Ref & Ref \\
\hline Female & $1.05(1.02,1.08)$ & $1.12(1.07,1.16)$ & $0.83(0.77,0.89)$ & $1.36(1.17,1.58)$ \\
\hline \multicolumn{5}{|l|}{ Ethnicity } \\
\hline Persian & Ref & Ref & - & - \\
\hline Non Persian & $1.03(1.00,1.05)$ & $1.03(1.00,1.06)$ & - & - \\
\hline Education $^{* * *}$ & $1.23(1.20,1.26)$ & $1.36(1.32,1.41)$ & $1.43(1.33,1.54)$ & $0.35(0.30,0.41)$ \\
\hline \multicolumn{5}{|l|}{ Brushing frequency } \\
\hline Daily & Ref & Ref & Ref & Ref \\
\hline Less than daily & $1.10(1.08,1.13)$ & $1.06(1.03,1.10)$ & $1.78(1.66,1.91)$ & $1.22(1.06,1.41)$ \\
\hline Cigarette ever used & $1.10(1.05,1.15)$ & $1.17(1.10,1.25)$ & - & - \\
\hline Cigarette current use & $1.14(1.09,1.19)$ & $1.14(1.06,1.21)$ & $1.94(1.74,2.15)$ & $0.75(0.61,0.93)$ \\
\hline Opium ever used & $1.17(1.12,1.22)$ & $1.23(1.16,1.30)$ & $1.59(1.40,1.82)$ & - \\
\hline Tobacco ever used & $1.07(1.05,1.09)$ & $1.11(1.08,1.15)$ & $1.16(1.08,1.24)$ & $0.60(0.52,0.70)$ \\
\hline \multicolumn{5}{|l|}{ Physical activity } \\
\hline Low & - & - & Ref & Ref \\
\hline Medium & - & - & $1.07(0.99,1.16)$ & $0.93(0.79,1.08)$ \\
\hline High & - & - & $1.09(1.27,1.50)$ & $0.75(0.63,0.89)$ \\
\hline \multicolumn{5}{|l|}{ Socioeconomic status } \\
\hline High & Ref & Ref & Ref & Ref \\
\hline Middle-high & $1.03(1.00,1.06)$ & $1.06(1.02,1.11)$ & $1.20(1.10,1.31)$ & $0.49(0.41,0.58)$ \\
\hline Middle-low & $1.05(1.02,1.09)$ & $1.09(1.04,1.14)$ & $1.40(1.29,1.53)$ & $0.32(0.26,0.38)$ \\
\hline Low & $1.07(1.03,1.10)$ & $1.10(1.06,1.15)$ & $1.54(1.41,1.69)$ & $0.19(0.16,0.24)$ \\
\hline
\end{tabular}

DMFT, missing and filled teeth.

"Data are presented as adjusted IRR (CI), IRR: incidence rate ratio, $\mathrm{Cl}$ : confidence interval, table of crude IRR is shown in the appendix.

"*The model for variables related to decayed and filled teeth is applied based on individual's existing teeth.

${ }^{* * *}$ For each lower level of education IRR is written (being illiterate vs. less than diploma vs. university degree).

and pathological abrasions more often occur in calcified dental tissues with increasing age. ${ }^{26}$ Besides, elderly people commonly use many medications due to different chronic conditions and diseases which result in dry mouth and hyposalivation and consequently trigger periodontal diseases and dental caries, the main causes of tooth extraction..$^{21,27}$

Rates of tooth loss were higher in Turks and minor ethnicities in our study. A higher rate of people of nonPersian ethnicity did not brush their teeth compared to people of Persian ethnicity $(55.9 \%$ vs. $44.1 \%, P<0.001)$. A significant difference has been reported in terms of oral health between the dominant ethnicity and minor ethnicities in other regions of the world, as well. ${ }^{28}$ This may be due to the difference in medical insurance and different cultures and habits related to oral hygiene.

Reports have shown that being married is protective against tooth loss and periodontitis. Married people have more dental cleanings and significantly more dental visits than unmarried people. ${ }^{29,30}$ However, we did not find any association between tooth loss or DMFT and marital status in our study.

Smokers in our study had more tooth loss (IRR: 1.17) and dental caries (IRR: 1.13), which is similar to other reports. ${ }^{31,32}$ According to a cross-sectional study in Iran, among a group of participants who were opiate dependent, none of the participants had a healthy periodontium. ${ }^{33}$ Besides the direct effects of drugs on oral health, individuals who have some form of drug dependency have many unhealthy behaviors including personal neglect of oral health care, high carbohydrate consumption and low priority for oral hygiene. Furthermore, drugs may suppress symptoms of tooth decay. ${ }^{34}$ In another study by Reddy et al, it was shown that substance abusers had more missing teeth, periodontal destruction and gingival recession. ${ }^{35}$ These studies were consistent with our results as we found that people who consumed opium had higher DMFT and more tooth loss.

In our study, individuals with higher physical activity had more tooth decay (IRR: 1.09). This could be 
explained as acute dehydration due to exercise and heat stress reduces saliva flow rate and causes saliva protein density and osmolality to increase. ${ }^{36}$ In one study, Ferreira et al evaluated the effects of endurance training on dental erosion, caries and saliva. They found that one hour per week increase in endurance training was correlated with a rise in DMFT at 0.24 points. ${ }^{37}$ This may be due to the fact that saliva protects against dental erosions due to its buffering and neutralizing characteristics on acidic food. ${ }^{38}$ However, we did not find any association between physical activity and tooth loss.

We found a higher prevalence of tooth loss and dental caries among illiterate participants which is similar to other reports; illiterate people or those with lower educational levels paid less attention to their oral health and had lower rates of visits to the dentists compared to people who finished high school or had higher education. ${ }^{28,37,38}$

Multiple studies have shown that lower SES is significantly associated with a higher prevalence of tooth loss and dental caries, ${ }^{25,39-41}$ which is similar to our results. Furthermore, people in lower socioeconomic conditions prefer extraction rather than restoration of teeth. ${ }^{25}$

This study has several limitations and strengths. This is a cross-sectional study and is limited by its nature and design. Causal relationships cannot be accurately assessed. Moreover, diagnosis of decay was based on clinical inspection and probe, and radiography images were not taken. One of the important strengths of this study is its large sample size with incorporation of a large variety of variables. Data were extracted from a population of individuals aged 40-75 years who belonged to a rural area which provides valuable information on the rural population of Iran. Tooth loss, dental caries and DMFT were assessed by trained physicians, which is much more reliable than individuals' self-report.

In conclusion, the present study showed that the prevalence of edentulism, partial edentulism and dental caries was high among the study population. The absence of large cohort studies focusing on oral health reinforces the demand for future studies. Some risk factors like cigarette and opium use, education and brushing of the teeth are modifiable; therefore, programs for increasing general awareness and improving healthy life style are needed. Furthermore, eradicating the causes of tooth loss and tooth decay should be established with special focus on older individuals, people with lower SES, illiterate participants, smokers and opiate consumers.

\section{Authors' Contribution}

RM, AG and HP aided in data gathering and design of the work. AS, $\mathrm{HM}, \mathrm{FS}$ and PA aided in analysis and interpretation of data for the work. SG aided in interpretation of results and preparation of the manuscript. All Authors aided critical revision of final manuscript. All authors have approved the final form of the manuscript.

\section{Conflict of Interest Disclosures}

The authors declare no conflict of interest related to this work.

\section{Ethical Statement}

The study protocol followed the guidelines reported in the declaration of Helsinki and was in accordance with the ethical standards of the institutional research committees of Shiraz and Tehran Universities of Medical Sciences. All participants gave their informed and written consent to enter the study.

\section{Acknowledgements}

The authors would like to thank all personnel who helped to establish this cohort registry and participants who patiently took part in this study. This study was registered as a dissertation for a Master of Public Health (MPH) degree by Saba Ghazimoghadam.

\section{Supplementary Materials}

Supplementary file 1 contains Tables S1- S2.

\section{References}

1. Glick M, Williams DM, Kleinman DV, Vujicic M, Watt RG, Weyant RJ. A new definition for oral health developed by the FDI World Dental Federation opens the door to a universal definition of oral health. Am J Orthod Dentofacial Orthop. 2016;221(12):792-3. doi: 10.1016/j.ajodo.2016.11.010.

2. Kassebaum N, Bernabé E, Dahiya M, Bhandari B, Murray C, Marcenes W. Global burden of severe tooth loss: a systematic review and meta-analysis. J Dent Res. 2014;93(7_suppl):20S8S. doi: 10.1177/0022034514537828.

3. Kassebaum N, Bernabé E, Dahiya M, Bhandari B, Murray C, Marcenes W. Global burden of untreated caries: a systematic review and metaregression. J Dent Res. 2015;94(5):650-8. doi: 10.1177/0022034515573272.

4. Petersen PE. World Health Organization global policy for improvement of oral health-World Health Assembly 2007. Int Dent J. 2008;58(3):115-21. doi: 10.1111/j.1875-595x.2008. tb00185.x.

5. Jahangiry L, Bagheri R, Darabi F, Sarbakhsh P, Sistani MMN, Ponnet K. Oral health status and associated lifestyle behaviors in a sample of Iranian adults: an exploratory household survey. BMC Oral Health. 2020;20(1):1-9. doi: 10.1186/s12903-02001072-z.

6. Shakeri R, Malekzadeh R, Etemadi A, Nasrollahzadeh D, Abedi-Ardekani B, Khoshnia M, et al. Association of tooth loss and oral hygiene with risk of gastric adenocarcinoma. Cancer Prev Res. 2013;6(5):477-82. doi: 10.1158/1940-6207.CAPR12-0491.

7. Holm-Pedersen P, Schultz-Larsen K, Christiansen N, Avlund K. Tooth loss and subsequent disability and mortality in old age. J Am Geriatr Soc. 2008;56(3):429-35. doi: 10.1111/j.15325415.2007.01602.x.

8. Petersen PE. Sociobehavioural risk factors in dental cariesinternational perspectives. Community Dent Oral Epidemiol. 2005;33(4):274-9. doi: 10.1111/j.1600-0528.2005.00235.x.

9. Morgano S, Doumit M, Shammari KA, Al-Suwayed A, AlSuwaidi A, Debaybo D, et al. Burden of oral disease in the Middle East: opportunities for dental public health. Int Dent J. 2010;60(3S1):197-9. doi: 10.1922/IDJ_2556Morgano03.

10. Asgari F, Majidi A, Koohpayehzadeh J, Etemad K, Rafei A. Oral hygiene status in a general population of Iran, 2011: a key lifestyle marker in relation to common risk factors of non-communicable diseases Int J Health Policy Manag. 2015;4(6):343. doi: 10.15171/ijhpm.2015.18.

11. Ahmadi A, Sahaf R, Rashedi V, Akbari Kamrani AA, Shati M, Delbari A. Relationship between oral health and demographic characteristics in retired elderly people in Iran. Iran J. Ageing. 2019;13(4):452-463. doi: 10.32598/SIJA.13.4.452.

12. Bongo AKS, Brustad M, Oscarson N, Jönsson B. Periodontal health in an indigenous Sámi population in Northern Norway: a cross-sectional study. BMC Oral Health. 2020;20(1):104. doi: 10.1186/s12903-020-01098-3. 
13. Carvalho JC, Schiffner U. Dental Caries in European Adults and Senior Citizens 1996-2016: ORCA Saturday Afternoon Symposium in Greifswald, Germany-Part II. Caries Res. 2019;53(3):242-52. doi: 10.1159/000492676.

14. Yuan JQ, Lv YB, Kraus VB, Gao X, Yin ZX, Chen HS, et al. Number of natural teeth, denture use and mortality in Chinese elderly: a population-based prospective cohort study. BMC Oral Health. 2020;20:1-11. doi: 10.1186/s12903-020-010849 .

15. Gandomkar A, Poustchi $H$, Moini $M$, Moghadami $M$, Imanieh $\mathrm{H}$, Fattahi MR, et al. Pars cohort study of non-communicable diseases in Iran: protocol and preliminary results. Int J Public Health. 2017;62(3):397-406. doi: 10.1007/s00038-016-08482 .

16. Molavi Vardajani $H$, Haghdoost AA, Shahravan A, Rad M. Cleansing and preparation of data for statistical analysis: A step necessary in oral health sciences research. J Oral Health Oral Epidemiol. 2016;5(4):171-185.

17. Adib A, Masoompour SM, Molavi Vardanjani H, Gondomkar A, Poustchi $\mathrm{H}$, Salehi A, et al. Smoking Water-Pipe, Opium Use and Prevalence of Heart Disease: A Cross-sectional Analysis of Baseline Data from the Pars Cohort Study, Southern Iran. Arch Iran Med. 2020;23(5):289-95. doi: 10.34172/aim.2020.17.

18. Kim Y, Park I, Kang M. Convergent validity of the international physical activity questionnaire (IPAQ): meta-analysis. Public Health Nutr. 2013;16(3):440-52. doi: 10.1017/ S1368980012002996.

19. Peltzer K, Hewlett S, Yawson AE, Moynihan P, Preet R, Wu F, et al. Prevalence of loss of all teeth (edentulism) and associated factors in older adults in China, Ghana, India, Mexico, Russia and South Africa. Int J Environ Res Public Health. 2014;11(11):11308-24. doi: 10.3390/ijerph111111308.

20. Dye BA, Thornton-Evans G, Li X, lafolla T. Dental caries and tooth loss in adults in the United States, 2011-2012. NCHS Data Brief. 2015;(197):197.

21. Raphael C. Oral health and aging. Am J Public Health. 2017;107(S1):S44-S45. doi: 10.2105/AJPH.2017.303835.

22. Gökalp S, Doğan B. Root caries in 35-44 and 65-74 year-olds in Turkey. Community Den Health. 2012;29(3):233-8.

23. Mamai-Homata E, Topitsoglou V, Oulis C, Margaritis V, Polychronopoulou A. Risk indicators of coronal and root caries in Greek middle aged adults and senior citizens. BMC Public Health. 2012;12(1):484. doi: 10.1186/1471-2458-12484.

24. Medina-Solís CE, Pérez-Núñez R, Maupomé G, CasanovaRosado JF. Edentulism among Mexican adults aged 35 years and older and associated factors. Am J Public Health. 2006;96(9):1578-81. doi: 10.2105/AJPH.2005.071209.

25. Vadavadagi SV, Srinivasa H, Goutham G, Hajira N, Lahari M, Reddy GP. Partial edentulism and its association with socio-demographic variables among subjects attending dental teaching institutions, India. J Int Oral Health. 2015;7(Suppl2):60-3.

26. Kunin AA, Evdokimova AY, Moiseeva NS. Age-related differences of tooth enamel morphochemistry in health and dental caries. EPMA Journal. 2015;6(1):3. doi: 10.1186/ s13167-014-0025-8

27. Fure S. Ten-year incidence of tooth loss and dental caries in elderly Swedish individuals. Caries Res. 2003;37(6):462-9. doi: 10.1159/000073401.

28. Kiyak HA, Reichmuth M. Barriers to and enablers of older adults' use of dental services. J Dent Educ. 2005;69(9):975-86.

29. Chattopadhyay A, Kumar JV, Green EL. The New York State minority health survey: determinants of oral health care utilization. J Public Health Dent. 2003;63(3):158-65. doi: 10.1111/j.1752-7325.2003.tb03494.x.

30. Buchwald S, Kocher T, Biffar R, Harb A, Holtfreter B, Meisel $\mathrm{P}$. Tooth loss and periodontitis by socio-economic status and inflammation in a longitudinal population-based study. J Clin Periodontol. 2013;40(3):203-11. doi: 10.1111/jcpe.12056.

31. Albandar JM, Streckfus CF, Adesanya MR, Winn DM. Cigar, pipe, and cigarette smoking as risk factors for periodontal disease and tooth loss. J Periodontol. 2000;71(12):1874-81. doi: 10.1902/jop.2000.71.12.1874.

32. Mai X, Wactawski-Wende J, Hovey KM, LaMonte MJ, Chen C, Tezal M, et al. Associations between smoking and tooth loss according to the reason for tooth loss: the Buffalo OsteoPerio Study. J Am Dent Assoc. 2013;144(3):252-65. doi: 10.14219/ jada.archive.2013.0112

33. Shekarchizadeh $H$, Khami MR, Mohebbi SZ, Ekhtiari $H$, Virtanen JI. Oral health status and its determinants among opiate dependents: a cross-sectional study. BMC Oral Health. 2019;19(1):5. doi: 10.1186/s12903-018-0691-3 .

34. Robinson P, Acquah S, Gibson B. Drug users: oral healthrelated attitudes and behaviors. Br Dent J. 2005;198(4):21924. doi: 10.1038/sj.bdj.4812090.

35. Reddy S, Kaul S, Agrawal C, Prasad M, Agnihotri J, Bhowmik N, et al. Periodontal status amongst substance abusers in Indian population. ISRN Dent. 2012;2012. doi: 10.5402/2012/460856.

36. Ferreira RdO, Corrêa MG, Magno MB, Almeida APCPSC, Fagundes NCF, Rosing CK, et al. Physical activity reduces the prevalence of periodontal disease: systematic review and meta-analysis. Front Physiol. 2019;10:234. doi: 10.3389/ fphys.2019.00234.

37. Castrejón-Pérez RC, Borges-Yáñez SA, Irigoyen-Camacho ME, Cruz-Hervert LP. Negative impact of oral health conditions on oral health related quality of life of community dwelling elders in Mexico city, a population based study. Geriatr Gerontol Int. 2017;17(5):744-752. doi: 10.1111/ggi.12780.

38. Shao R, Hu T, Zhong YS, Li X, Gao YB, Wang YF, et al. Socio-demographic factors, dental status and health-related behaviors associated with geriatric oral health-related quality of life in Southwestern China. Health Qual Life Outcomes. 2018;16(1):98. doi: 10.1186/s12955-018-0925-8.

39. Schwendicke F, Dörfer C, Schlattmann P, Page LF, Thomson W, Paris S. Socioeconomic inequality and caries: a systematic review and meta-analysis. J Dent Res. 2015;94(1):10-8. doi: 10.1177/0022034514557546.

40. Al-Hadi Hamasha A, Sasa I, Al Qudah M. Risk indicators associated with tooth loss in Jordanian adults. Community Dent Oral Epidemiol. 2000;28(1):67-72. doi: 10.1034/j.16000528.2000.280109.x.

41. Wang L, Cheng L, Yuan B, Hong X, Hu T. Association between socio-economic status and dental caries in elderly people in Sichuan Province, China: a cross-sectional study. BMJ Open. 2017;7(9):e016557. doi: 10.1136/bmjopen-2017-016557. 